\title{
COMPOSIÇÃO CORPORAL E EXIGÊNCIAS NUTRICIONAIS DE MAGNÉSIO, POTÁSSIO E SÓDIO DE CORDEIROS SANTA INÊS E SEUS CRUZAMENTOS COM BERGAMÁCIA, ILE DE FRANCE E TEXEL DOS 15 AOS 45 KG DE PESO VIVO ${ }^{1}$
}

\author{
Body composition and nutricional requeriments the magnesium, sodium and potassium \\ of lambs Santa Inês and their crossing with Bergamácia, Ile de France of texel
}

\author{
Edinéia Alves Moreira Baião², Juan Ramón Olalquiaga Perez ${ }^{3}$, Afranio Afonso Ferrari Baião², \\ Leonardo Alves Baião ${ }^{4}$, Luciana Castro Geraseev², Júlio César Teixeira ${ }^{2}$ \\ Ivo Francisco de Andrade ${ }^{3}$, André Nunes de Oliveira²
}

\begin{abstract}
RESUMO
O presente trabalho foi conduzido no setor de Ovinocultura do Departamento de Zootecnia da Universidade Federal de Lavras-MG, com o objetivo de determinar a composição corporal e estimar as exigências nutricionais dos macroelementos minerais Magnésio (Mg), Sódio (Na) e Potássio (K), para ganho de cordeiros em crescimento. Foram utilizados 48 cordeiros machos inteiros, sendo 12 Santa Inês X Santa Inês (SI X SI), 12 Bergamácia X Santa Inês (BE X SI), 12 Ile de France X Santa Inês (IF X SI) e 12 Texel X Santa Inês (TE X SI). Três animais de cada grupo genético foram abatidos no início do experimento para determinação das quantidades de $\mathrm{Mg}$, $\mathrm{Na}$ e $\mathrm{K}$ retidos em seus corpos, servindo como animais-referência para a técnica do abate comparativo. Os animais remanescentes de cada grupo genético foram confinados até atingirem os pesos pré-determinados para o abate de 25, 35 e $45 \mathrm{~kg}$ de peso vivo. A composição corporal em $\mathrm{Mg}$, $\mathrm{Na}$ e $\mathrm{K}$ foi estimada por meio de equações de predição, obtidas pela regressão do logaritmo da quantidade dos minerais presentes no corpo vazio em função do logaritmo do peso do corpo vazio (PCV). As exigências líquidas de $\mathrm{Mg}$, $\mathrm{Na}$ e $\mathrm{K}$ para o ganho em peso foram estimadas a partir da derivação das equações de predição da composição corporal. A composição corporal de $\mathrm{Mg}$ do grupo genético Santa Inês diferiu dos demais grupos, e para animais de 15 e $45 \mathrm{~kg}$ de peso vivo, o valor encontrado foi: 0,606 e 0,514 g de $\mathrm{Mg} / \mathrm{kg}$ de PCV para os animais puros Santa Inês e 0,524 e $0,475 \mathrm{~g}$ de $\mathrm{Mg} / \mathrm{kg}$ de PCV para os demais grupos genéticos. No caso do $\mathrm{Na}$ e $\mathrm{K}$ houve diferenças nas estimativas para o grupo genético IF X SI sendo os valores: 1,604 e 1,219 g de Na/kg de PCV e 1,938 e 1,592 g de K/kg de PCV, para os demais grupos genéticos, os valores foram: 1,665 e 1,270 g de Na/kg de PCV e 2,144 e 1,685 g de K/kg de PCV. As estimativas das exigências líquidas de $\mathrm{Mg}$, encontrada nesta pesquisa, foram: $0,40 \mathrm{~g}$ e $0,35 \mathrm{~g}$ de $\mathrm{Mg} / \mathrm{kg}$ de PV para animais puros Santa Inês e $0,38 \mathrm{~g}$ e $0,33 \mathrm{~g}$ de $\mathrm{Mg} / \mathrm{kg}$ de PV para os demais animais com 15 e $45 \mathrm{~kg}$ de peso vivo, respectivamente. As exigências líquidas de $\mathrm{K}$ e $\mathrm{Na}$ estimadas nesta pesquisa para animais de 15 e $45 \mathrm{~kg}$ de peso vivo: $1,30 \mathrm{~g}$ e 1,06 $\mathrm{g}$ de $\mathrm{K} / \mathrm{kg} \mathrm{de} \mathrm{PV}, 0,99 \mathrm{~g}$ e $0,75 \mathrm{~g}$ de Na/kg de PV para animais IF X SI e 1,37 g e 1,07 g de K/kg de PV e 1,03 g e 0,78 g de Na/kg de PV para os demais animais.
\end{abstract}

Termos para indexação: Composição corporal, magnésio, ovino, potássio, requerimento, sódio.

\section{ABSTRACT}

The present work was conducted in the Sheep Division of Animal Science Department at Universidade Federal de Lavras - M.G., aiming to determine the body composition of lambs and to estimate the nutritional requirements of the mineral macroelements Magnesium $(\mathrm{Mg})$, Sodium $(\mathrm{Na})$ and Potassium $(\mathrm{K})$ for weight gain of growing lambs. It was used 48 not castrated male lambs, being 12 Santa Inês X Santa Inês (SI X SI), 12 Bergamácia X Santa Inês (BE X SI), 12 Ile de France X Santa Inês (IF X SI) and 12 Texel X Santa Inês (TE X SI). For determination of Mg, Na and K amounts kept in the animal body, three animals of each genetic group were slaughtered in the beginning of the experiment making as reference animals for the comparative slaughtered technique. The remaining animals of each genetic group were confined until the slaughter where they reached 25, 35 and $45 \mathrm{~kg}$ of live weight. The body composition in $\mathrm{Mg}$, $\mathrm{Na}$ and $\mathrm{K}$ was estimated by the prediction equations

(Recebido para publicação em 22 de novembro de 2002 e aprovado em 24 de julho de 2003)

\footnotetext{
1. Extraído da dissertação de mestrado apresentada pelo primeiro autor à Universidade Federal de Lavras/UFLA - Caixa Postal 37 - $37200-000$ - Lavras, MG.

2. Alunos do Curso de Pós-Graduação em Zootecnia/UFLA. eambaiao@ yahoo.com.br

3. Professores do Departamento de Zootecnia da UFLA.

4. Aluno do curso de Graduação em Ciências Biológicas da UFES.
} 
obtained by the regression of the minerals amount logarithm in the empty body in function of the empty body weight logarithm (EBW). The net requirements of $\mathrm{Mg}, \mathrm{Na}$ and $\mathrm{K}$ for the weight gain were estimated from the derivation of the prediction equations of the body composition. The body composition of $\mathrm{Mg}$ of genetic grups SI differed of the other geneticgrups and the values varied per kilo of empty body weight (EBW) for animals from 15 and $45 \mathrm{~kg}$ of live weight was: 0,606 and 0,514 g of $\mathrm{Mg} / \mathrm{kg}$ of EBW for the Santa Inês pure animals and 0,524 and 0,475 g of Mg/kg of EBW for the other genetic groups. In the case of the Na and $\mathrm{K}$ there were differences in the estimates for the IF X SI genetic group and the values varied from 1,604 and 1,219 $\mathrm{g}$ of $\mathrm{Na} / \mathrm{kg}$ of EBW and from 1,938 and 1,592 $\mathrm{g}$ of $\mathrm{K} / \mathrm{kg}$ of EBW; for the other genetic groups the values varied from 1,665 and $1,270 \mathrm{~g}$ of Na/kg of EBW and 2,144 and 1,685 g of K/ kg of EBW. The net requirement of $\mathrm{Mg}$, estimate in this research were: $0,40 \mathrm{~g}$ and $0,35 \mathrm{~g}$ of $\mathrm{Mg} / \mathrm{kg}$ of LW for Santa Inês pure animals and $0,38 \mathrm{~g}$ and $0,33 \mathrm{~g} \mathrm{of} \mathrm{Mg} / \mathrm{kg}$ of LW for the other animals with 15 and $45 \mathrm{~kg}$ of live weight. The net requirement of $\mathrm{K}$ and $\mathrm{Na}$ for crossbred animals IF X SI differed from the requirements of the other genetic groups animals. The following values for animals of 15 and $45 \mathrm{~kg}$ of live weight were:1,30 $\mathrm{g}$ and 1,06 $\mathrm{g}$ of $\mathrm{K} / \mathrm{kg}$ of LW, $0,99 \mathrm{~g}$ and $0,75 \mathrm{~g}$ of Na/kg of LW for IF X SI animals and 1,37 $\mathrm{g}$ and 1,07 $\mathrm{g}$ of $\mathrm{K} / \mathrm{kg}$ of LW and from 1,03 $\mathrm{g}$ and $0,78 \mathrm{~g}$ of $\mathrm{Na} / \mathrm{kg}$ of $\mathrm{LW}$ for the other animals.

Index terms: Body composition, magnesium, sheep, potassium, requirement, sodium.

\section{INTRODUÇÃO}

Os elementos minerais constituem cerca de $5 \%$ do peso vivo do animal e dieteticamente são essenciais para os ruminantes e para os microorganismos presentes no rúmen e no intestino, exercendo influência direta e indireta sobre o crescimento, engorda, produção de leite, reprodução, produção de lã em ovinos e manutenção dos processos vitais.

O magnésio exerce funções essenciais ligadas aos sistemas enzimáticos particularmente, aquele do metabolismo dos carboidratos e lipídeos, é requerido na oxidação celular e exerce grande influência na atividade neuromuscular. Já o potássio e o sódio são essenciais à vida, tendo como principais funções a regulação do balanço osmótico celular, o equilíbrio ácido-base e atuam em vários sistemas enzimáticos e balanço hídrico do organismo (MCDOWELL, 1999).

Uma suplementação mineral adequada é ainda mais importante, tendo em vista o empobrecimento dos solos, resultando em forrageiras deficientes em um grande número de macro e microelementos minerais responsáveis diretos pela perda de peso, diarréia, anemia, perda de apetite, e anormalidade óssea entre outros problemas (MCDOWELL, 1999).

Apesar das dificuldades encontradas na determinação das exigências minerais para ovinos, em razão de fatores que influenciam negativamente, como: disponibilidade e qualidade dos alimentos, raça, sexo, peso e condições climáticas, alguns experimentos têm sido realizados com a finalidade de definir tais exigências.

O ARC (1980) estabeleceu modelos matemáticos que permitem estimar a composição mineral do corpo e, conseqüentemente, a exigência líquida para o ganho em ruminantes de diferentes raças, idades e pesos. O ARC (1980), considerando ovinos em crescimento, quantifi- cou os conteúdos corporais de magnésio, potássio e sódio em $0,41,1,8$ e $1,1 \mathrm{~g} / \mathrm{kg}$ de peso corporal vazio $(\mathrm{PCV})$, respectivamente.

No Brasil, as exigências nutricionais ainda são pouco estudadas e o cálculo de rações baseia-se em tabelas internacionais (SILVA, 1995). Em virtude da diversidade entre as condições climáticas, raça, idade do animal, disponibilidade e qualidade de alimentos, verificadas no Brasil, em comparação às de outros países, ocorrem níveis de performance animal diferentes do esperado.

Portanto, torna-se necessário o estudo da eficiência da utilização dos nutrientes pelos ovinos lanados e deslanados criados no Brasil e de suas exigências, uma vez que essas tabelas utilizam animais diferentes dos nossos e sob condições climáticas diversaConduziu-se este trabalho com o objetivo de determinar a composição corporal e estimar as exigências nutricionais em magnésio, potássio e sódio de animais puros Santa Inês (SI) e mestiços Bergamácia (BE), Ilê de France (IF) e Texel (TE), criados na região sul de Minas Gerais.

\section{MATERIAL E MÉTODOS}

O trabalho foi conduzido no Setor de Ovinocultura do Departamento de Zootecnia da Universidade Federal de Lavras, em Lavras, MG. Foram utilizados 48 cordeiros machos inteiros procedentes dos cruzamentos SI X SI; BE X SI; IF X SI; TE X SI com peso vivo inicial de $15 \mathrm{~kg}$.

No experimento, os animais foram divididos em 4 grupos: 12 animais, escolhidos aleatoriamente, sendo três de cada grupo genético, foram sacrificados no início do trabalho para avaliação do conteúdo de magnésio, potássio e sódio corporais, servindo como animais de referência para o método de abate comparativo. Os 
animais remanescentes foram colocados em gaiolas individuais, onde receberam alimentação à vontade. A dieta experimental (Tabela 1) foi balanceada, de forma a atender às exigências nutricionais de proteína, energia e minerais, segundo as recomendações do ARC (1980), sendo fornecida duas vezes ao dia, às 8 e às 16 horas. $O$ controle do desenvolvimento dos animais foi feito por meio de pesagens semanais, que foram efetuadas na parte da manhã, antes do animal receber a alimentação diária.

Ao atingirem os pesos pré-determinados de 15, 25,35 e $45 \mathrm{~kg}$ de peso vivo, os animais foram abatidos com corte da carótida e da jugular, sendo submetidos a um jejum prévio de 16 horas, com acesso à água. No abate, determinou-se o peso corporal vazio, subtraindose do peso vivo o peso do conteúdo gastrointestinal, vesícula biliar e bexiga.

Para obtenção das amostras referentes ao corpo dos animais, todo o corpo do animal (carcaça, pele, vísceras, sangue, patas e cabeça) foi congelado, sendo esse material, posteriormente, cortado em uma serra de fita e moído em cutter de 30 HP e 1775 rpm. Posteriormente, todos os procedimentos citados acima foram novamente repetidos para, então, serem retiradas as amostras para as análises químicas. As análises químicas das amostras do corpo dos animais foram obtidas pela digestão nitroperclórica, obtendo-se, dessa forma, a solução mineral. Desse extrato, foram feitas diluições para determinação dos diferentes minerais em estudo.

Para o magnésio, as diluições foram obtidas adicionando-se cloreto de estrôncio, e as leituras, tomadas em espectrofotômetro de absorção atômica. Para as diluições do sódio e potássio, foi utilizado o nitrato de lítio, e as leituras, tomadas em espectrofotômetro de chama.

As concentrações corporais dos minerais foram determinadas em função dos conteúdos percentuais desses nas amostras do corpo dos animais. Com base nesses dados, foram obtidas equações de predição da composição corporal em termos de macrominerais.

As equações de predição do conteúdo corporal de magnésio, sódio e potássio foram obtidas por meio da regressão do logaritmo da quantidade do nutriente no corpo vazio em função do logaritmo do peso do corpo vazio (ARC, 1980).

TABELA 1 - Teores de matéria seca (MS), energia metabolizável (EM), proteína bruta (PB) e composição em minerais da dieta experimental.

\begin{tabular}{|c|c|c|c|c|c|c|}
\hline Ingredientes & $\begin{array}{l}\text { MS }^{1} \\
(\%)\end{array}$ & $\begin{array}{c}\mathrm{EM}^{2} \\
\text { (Kcal/kg) }\end{array}$ & $\begin{array}{l}\mathbf{P B}^{1} \\
(\%)\end{array}$ & $\begin{array}{l}\mathbf{M g}^{1} \\
(\%)\end{array}$ & $\begin{array}{l}\mathrm{Na}^{1} \\
(\%)\end{array}$ & $\begin{array}{c}\mathbf{K}^{1} \\
(\%)\end{array}$ \\
\hline Milho moido & 66,23 & 2087 & 6,49 & 0,062 & 0,025 & 0,233 \\
\hline Farelo de soja & 12,37 & 394 & 6,28 & 0,031 & 0,004 & 0,225 \\
\hline Feno de coast cross & 20,25 & 395 & 2,44 & 0,041 & 0,009 & 0,363 \\
\hline Calcário cálcitico & 0,85 & & & & & \\
\hline Sal comum & 0,25 & & & & 0,094 & \\
\hline Supl.microminerais ${ }^{3}$ & 0,01 & & & & & \\
\hline Supl. Vitamínico ${ }^{3}$ & 0,04 & & & & & \\
\hline TOTAL & 100,00 & 2876 & 15,21 & 0,134 & 0,132 & 0,821 \\
\hline
\end{tabular}

1'Análises realizadas no laboratório de Nutrição Animal do Departamento de Zootecnia

2NRC (1985)

${ }^{3}$ Suplemento Microminerais e Vitamínico (nutriente/Kg de suplemento): vit. A 2.500.000 UI, Vit. D3 500.000UI, Vit. E $3000 \mathrm{mg}$, Tiamina $750 \mathrm{mg}$, Riboflavina $1000 \mathrm{mg}$, VitB12 $2800 \mathrm{mcg}$, Niacina $500 \mathrm{mg}$, Selênio $150 \mathrm{mg}$, Iodo $1000 \mathrm{mg}$, Cobalto $600 \mathrm{mg}$, Ferro $35000 \mathrm{mg}$, Cobre $20000 \mathrm{mg}$, Manganês $49000 \mathrm{mg}$, Zinco 75000 mg.

$$
\log y=a+b \log x \quad \text { Em que: }
$$


Log y = Logaritmo do conteúdo total do macromineral no corpo vazio

$\mathrm{a}=$ intercepto

$\log x=$ Logaritmo do peso corporal vazio

$\mathrm{b}=$ Coeficiente de regressão do conteúdo do macromineral em função do peso corporal vazio.

Foi realizada uma análise de comparação de equações lineares (Snedecor \& Cochran, 1967) entre as equações de predição estimadas com todos os animais, para detectar possíveis diferenças entre os grupos genéticos.

As exigências líquidas para o ganho em peso corporal vazio (PCV) foram estimadas pela derivação das equações de predição corporal, obtendo-se equações do tipo $\mathrm{y}^{\prime}=10^{\mathrm{a}} \times \mathrm{b} \times \mathrm{PCV}^{(\mathrm{b}-1)}$. As exigências líquidas desses minerais para o ganho de peso vivo foram calculadas dividindo-se as exigências líquidas de ganho corporal vazio pelo fator 1,25 , calculado a partir das equações de conversão do peso corporal vazio em peso vivo. Para as exigências líquidas de mantença, foram utilizados os valores propostos pelo ARC (1980).

Para obtenção das exigências dietéticas totais, foram utilizados os valores de perdas endógenas de 3,0 $\mathrm{mg}$ e $25,8 \mathrm{mg} / \mathrm{kg}$ PV e de disponibilidade biológica de 17 e $91 \%$, para o magnésio e o sódio, recomendados pelo (ARC, 1980). Já as perdas endógenas totais do potássio consideradas foram: perda fecal $(1,0 \mathrm{~g} / \mathrm{kg}$ MS consumida), urinária $(37,5 \mathrm{mg} / \mathrm{kg} \mathrm{PV} /$ dia $)$, pela saliva (7,0 $\mathrm{mg} / \mathrm{kg}$ PV dia) e pela pele $(0,1 \mathrm{~g} / \mathrm{dia})$, sendo a disponibilidade considerada de $100 \%$ (ARC, 1980).

\section{RESULTADOS E DISCUSSÃO}

Na Tabela 2 estão apresentados os resultados médios da composição corporal em magnésio, potássio e sódio encontrados para os animais SI e mestiços BE, IF e TE em diferentes pesos vivos (15, 25, 35 e $45 \mathrm{~kg})$.

Quanto à concentração corporal de magnésio, potássio e sódio dos cordeiros SI e mestiços BE, IF e TE, observa-se que ocorreu decréscimo nas concentrações desses minerais com o aumento do peso vivo, o que pode ser explicado pelo aumento no teor de gordura corporal. Já para o magnésio, a possível explicação é a redução na proporção de ossos na carcaça à medida que aumentou o peso vivo dos cordeiros, pois aproximadamente $70 \%$ de magnésio estão presentes nos ossos.

Outros autores também estimaram valores decrescentes para o conteúdo corporal de magnésio,

TABELA 2 - Valores médios da composição corporal de cordeiros Santa Inês, Bergamacia X Santa Inês, Ile de France X Santa Inês e Texel X Santa Inês abatidos em diferentes pesos vivos. potássio e sódio (GERASEEV et al., 2001; TRINDADE et al., 2000; GRACE, 1983; ANNENKOV, 1982). Entretanto, o ARC (1980) considera os valores fixos das concentrações desses minerais de $0,41 \mathrm{~g}$ de magnésio, $1,8 \mathrm{~g}$ de potássio e $1,1 \mathrm{~g}$ de $\mathrm{Na} / \mathrm{kg}$ de PCV.

Os valores estimados do conteúdo de potássio encontrados nesta pesquisa para cordeiros SI e mestiços $\mathrm{BE}$, IF e TE foram diferentes dos estimados pelo ARC (1980). Para o sódio, os valores estimados nesta pesquisa foram superiores aos estimados pelo ARC (1980). Isso pode indicar diferenças na proporção de músculos, ossos e gordura nos animais utilizados neste estudo, quando comparados com os animais utilizados pelo ARC (1980).

Baseando-se na composição corporal dos animais e seus respectivos peso vivo e peso corporal vazio, foram determinadas as equações de predição da composição corporal (Tabela 3) para esses minerais para os quatro grupos genéticos estudados (SI, BE, IF, TE), com pesos variando dos 15 aos $45 \mathrm{~kg}$ de peso vivo, em que foi aplicada a análise de comparação de equações lineares (SNEDECOR e COCHRAN, 1967), que mostrou não haver diferença significativas para os grupos BE, IF e TE para estimar as quantidades de magnésio no peso corporal vazio. Assim, adotou-se uma equação geral obtida com todos os animais do experimento. Já para o grupo genético Santa Inês, detectou-se diferença para o mineral magnésio $(\mathrm{P}<0,05)$; logo, adotou-se uma equação específica. Para o potássio e sódio, foram detectadas diferenças significativas para o grupo genético IF, enquanto os outros grupos genéticos não mostraram diferença significativas $(\mathrm{P}>0,05)$. Dessa forma, foram adotadas equações específicas para estimar os valores de potássio e sódio para os animais IF, e equações gerais, para os outros grupos genéticos.

Os valores dos coeficientes de determinação encontrados para as equações foram significativos $(\mathrm{P}<0,05)$ e mostram que houve um bom ajustamento, com baixa dispersão dos dados em torno da linha de regressão. A composição do corpo vazio em magnésio, potássio e sódio, em função do peso vivo, foi estimada a partir das equações gerais e equações específicas (SI e IF), para todos os animais estudados.

$\mathrm{Na}$ Tabela 4 são apresentadas as estimativas do conteúdo de magnésio, potássio e sódio por quilo de peso corporal vazio. 


\begin{tabular}{|c|c|c|c|c|}
\hline \multirow[b]{2}{*}{ Item } & \multicolumn{4}{|c|}{ Santa Inês } \\
\hline & $15 \mathrm{~kg}$ & $25 \mathrm{~kg}$ & $35 \mathrm{~kg}$ & $45 \mathrm{~kg}$ \\
\hline & & Composição & Corporal & \\
\hline M.S. (\%) & $37,94 \pm 2,663$ & $41,57 \pm 0,804$ & $42,88 \pm 0,825$ & $45,05 \pm 0,937$ \\
\hline Gordura (\%MN) & $10,15 \pm 0,473$ & $14,16 \pm 0,107$ & $19,09 \pm 0,268$ & $22,16 \pm 2,057$ \\
\hline Mgnésio (\%MN) & $0,058 \pm 0,011$ & $0,056 \pm 0,005$ & $0,052 \pm 0,005$ & $0,049 \pm 0,003$ \\
\hline Potássio (\%MN) & $0,207 \pm 0,020$ & $0,190 \pm 0,08$ & $0,174 \pm 0,006$ & $0,162 \pm 0,004$ \\
\hline \multirow[t]{3}{*}{ Sódio (\%MN) } & $0,158 \pm 0,013$ & $0,141 \pm 0,006$ & $0,131 \pm 0,002$ & $0,123 \pm 0,003$ \\
\hline & \multicolumn{4}{|c|}{ Bergamácia } \\
\hline & & Composição & Corporal & \\
\hline M.S. (\%) & $38,41 \pm 1,226$ & $41,33 \pm 1,079$ & $42,71 \pm 0,705$ & $44,37 \pm 1,406$ \\
\hline Gordura(\%MN) & $10,31 \pm 0,430$ & $14,01 \pm 0,282$ & $18,95 \pm 0,514$ & $22,07 \pm 0,836$ \\
\hline Magnésio(\%MN) & $0,055 \pm 0,010$ & $0,056 \pm 0,012$ & $0,050 \pm 0,003$ & $0,048 \pm 0,002$ \\
\hline Potasio(\%MN) & $0,210 \pm 0,019$ & $0,195 \pm 0,023$ & $0,187 \pm 0,010$ & $0,166 \pm 0,006$ \\
\hline \multirow[t]{2}{*}{ Sodio(\%MN) } & $0,162 \pm 0,012$ & $0,148 \pm 0,008$ & $0,145 \pm 0,009$ & $0,124 \pm 0,003$ \\
\hline & \multicolumn{4}{|c|}{ ILE DE FRANCE } \\
\hline \multirow[t]{2}{*}{ Item } & $15 \mathrm{~kg}$ & $25 \mathrm{~kg}$ & 35 kg & $45 \mathrm{~kg}$ \\
\hline & & Composição & Corporal & \\
\hline M. S. (\%) & $38,91 \pm 0,944$ & $41,97 \pm 0,229$ & $43,03 \pm 1,664$ & $47,24 \pm 0,783$ \\
\hline Gordura $(\% \mathrm{MN})$ & $11,48 \pm 0,445$ & $14,41 \pm 0,197$ & $19,12 \pm 0,034$ & $24,26 \pm 0,343$ \\
\hline Magnésio (\%MN) & $0,051 \pm 0,008$ & $0,050 \pm 0,006$ & $0,046 \pm 0,007$ & $0,045 \pm 0,003$ \\
\hline Potássio (\%MN) & $0,196 \pm 0,005$ & $0,172 \pm 0,003$ & $0,169 \pm 0,009$ & $0,163 \pm 0,009$ \\
\hline \multirow[t]{3}{*}{ Sódio (\%MN) } & $0,151 \pm 0,011$ & $0,146 \pm 0,012$ & $0,125 \pm 0,006$ & $0,122 \pm 0,010$ \\
\hline & \multicolumn{4}{|c|}{ TEXEL } \\
\hline & & Composição & Corporal & \\
\hline M.S. ( \%) & $38,73 \pm 1,626$ & $41,81 \pm 0,394$ & $42,67 \pm 0,446$ & $45,58 \pm 0,357$ \\
\hline Gordura (\%MN) & $11,45 \pm 0,240$ & $14,18 \pm 0,858$ & $19,07 \pm 0,297$ & $23,99 \pm 1,018$ \\
\hline Magnésio (\%MN) & $0,049 \pm 0,023$ & $0,048 \pm 0,004$ & $0,047 \pm 0,008$ & $0,046 \pm 0,003$ \\
\hline Potássio(\%MN) & $0,199 \pm 0,020$ & $0,186 \pm 0,004$ & $0,178 \pm 0,019$ & $0,164 \pm 0,003$ \\
\hline $\operatorname{Sodio}(\% \mathrm{MN})$ & $0,158 \pm 0,023$ & $0,141 \pm 0,003$ & $0,138 \pm 0,011$ & $0,123 \pm 0,004$ \\
\hline
\end{tabular}


TABELA 3 - Equações de regressão para o peso de corpo vazio (g) em função do peso vivo(g) e para a quantidade corporal de magnésio, potássio e sódio presentes no corpo vazio, em função do peso corporal vazio em cordeiros SI e mestiços BE, IF e TE dos 15 aos $45 \mathrm{~kg}$ de PV.

\begin{tabular}{|c|c|c|}
\hline Item & Equação & $\mathbf{R}^{2}$ \\
\hline Peso corp. Vazio(g) & $-1852,059+0,848100 \mathrm{PV}$ & 96,79 \\
\hline Magnésio(g) SI & $\log \mathrm{Mg}=-2,66026+0,862070 \log \mathrm{PCV}$ & 92,05 \\
\hline Magnésio(g) BE,IF,TE & $\log \mathrm{Mg}=-2,95142+0,918429 \log \mathrm{PCV}$ & 84,79 \\
\hline Potássio(g) IF & $\log K=-2,05630+0,837360 \log P C V$ & 98,05 \\
\hline Potássio(g) SI, BE, TE & $\log K=-1,86117+0,799933 \log P C V$ & 96,57 \\
\hline Sódio(g) IF & $\log \mathrm{Na}=1,87483+0,772119 \log \mathrm{PCV}$ & 95,56 \\
\hline Sódio(g) SI, BE, TE & $\log \mathrm{Na}=-1,8755+0,776225 \log \mathrm{PCV}$ & 96,92 \\
\hline
\end{tabular}

TABELA 4 - Estimativa do conteúdo de magnésio, potássio e sódio no corpo vazio em função do peso corporal vazio em cordeiros SI, e mestiços BE, IF e TE.

\begin{tabular}{|c|c|c|c|c|c|c|c|}
\hline \multirow{2}{*}{$\begin{array}{l}\text { PV } \\
(\mathrm{kg})\end{array}$} & \multirow{2}{*}{$\begin{array}{r}\text { PCV } \\
(\mathrm{kg})\end{array}$} & \multicolumn{6}{|c|}{ Nutriente (g / kg PCV) } \\
\hline & & $\begin{array}{l}\text { Mg } \\
\text { (SI) }\end{array}$ & $\begin{array}{c}\text { Mg } \\
(\mathbf{B E}, \mathrm{IF}, \mathrm{TE})\end{array}$ & $\begin{array}{l}\mathrm{Na} \\
\text { (IF) }\end{array}$ & $\begin{array}{l}\mathbf{K} \\
(\mathbf{I F})\end{array}$ & $\begin{array}{c}\mathrm{Na} \\
(\mathrm{SI}, \mathrm{BE}, \mathrm{TE})\end{array}$ & $\begin{array}{c}\mathrm{K} \\
(\mathrm{SI}, \mathrm{BE}, \mathrm{TE})\end{array}$ \\
\hline 15 & 10,87 & 0,606 & 0,524 & 1,604 & 1,938 & 1,665 & 2,144 \\
\hline 20 & 15,10 & 0,580 & 0,510 & 1,489 & 1,837 & 1,546 & 2,007 \\
\hline 25 & 19,35 & 0,560 & 0,500 & 1,407 & 1,764 & 1,463 & 1,910 \\
\hline 30 & 23,60 & 0,545 & 0,492 & 1,345 & 1,708 & 1,400 & 1,836 \\
\hline 35 & 27,83 & 0,533 & 0,485 & 1,295 & 1,662 & 1,349 & 1,777 \\
\hline 40 & 33,92 & 0,519 & 0,478 & 1,238 & 1,610 & 1,290 & 1,708 \\
\hline 45 & 36,31 & 0,514 & 0,475 & 1,219 & 1,592 & 1,270 & 1,685 \\
\hline Média & & 0,551 & 0,494 & 1,371 & 1,730 & 1,426 & 1,866 \\
\hline $\mathrm{RC}(1980)$ & & 0,41 & 0,41 & 1,1 & 1,8 & 1,1 & 1,8 \\
\hline
\end{tabular}

O ARC (1980) preconiza valores fixos de composição do ganho de $0,41 \mathrm{~g}, 1,8 \mathrm{~g}$ e $1,1 \mathrm{~g}$ para o magnésio, potássio e sódio, respectivamente.

Assim como ocorreu com a composição corporal, os valores de composição do ganho encontrados neste trabalho diferiram dos valores fixos apresentados pelo ARC (1980); os valores médios de magnésio para os quatro grupos genéticos deste trabalho foram maiores, os do potássio, inferiores, e os de sódio, semelhantes aos valores preconizados pelo ARC (1980).
As quantidades de sódio estimadas variaram de $1,29 \mathrm{~g}$ a $0,98 \mathrm{~g}$ de $\mathrm{Na}$ por $\mathrm{kg}$ de PCV, para animais SI, $\mathrm{BE}$ e TE, quando o PV passou de 15 para $45 \mathrm{~kg}$. Esses valores são 17,27\% superiores e 12,24 \% inferiores ao valor fixo de $1,1 \mathrm{~g}$ de $\mathrm{Na}$ por $\mathrm{kg}$ de $\mathrm{PCV}$ recomendado pelo ARC (1980).

É possível que essas diferenças na composição do ganho em magnésio, potássio e sódio são devidas às diferenças existentes na proporção de ossos e gordura corporal. Portanto, é possível que fatores como raça, peso e condições climáticas alterem as concentrações corporais desses minerais. 
Quanto ao conteúdo de magnésio nos animais Santa Inês, observou-se uma variação de 0,606 a 0,514 g para os pesos vivos de 15 a $45 \mathrm{~kg}$, respectivamente. Esses valores estão próximos dos valores estimados por Geraseev et al. (2001), e superiores aos estimados por Trindade et al. (2000), para dois grupos raciais deslanados e lanados.

Para a predição da composição do ganho em magnésio, potássio e sódio, derivaram-se às equações de predição da composição corporal, obtendo-se, então, equações que permitiram estimar as exigências líquidas desses minerais para o ganho em peso corporal vazio:

\begin{tabular}{|c|c|c|}
\hline Magnésio (g) & SI & $Y^{\prime}=0,0018349 \cdot P_{C V}^{-0,13793}$ \\
\hline Magnésio (g) & BE, IF e TE & $\mathrm{Y}^{\prime}=0,001027 \cdot \mathrm{PCV}^{-0,081971}$ \\
\hline Potássio (g) & IF & $\mathrm{Y}^{\prime}=0,0073555 \cdot \mathrm{PCV}^{-0,162648}$ \\
\hline Potássio (g) & SI, BE e TE & $\mathrm{Y}^{\prime}=0,011012 \cdot \mathrm{PCV}^{-0,200067}$ \\
\hline Sódio $(\mathrm{g})$ & IF & $\mathrm{Y}^{\prime}=0,0103 . \mathrm{PCV}^{-0,227881}$ \\
\hline Sódio (g) & SI, BE e TE & $Y^{\prime}=0,010339 \cdot P_{C V}^{-0,223775}$ \\
\hline
\end{tabular}

Na Tabela 5 são mostrados os valores da estimativa da concentração de magnésio, potássio e sódio corporal do ganho em peso de corpo vazio de cordeiros SI e mestiços BE, IF e TE, em função do peso corporal vazio.

As estimativas das exigências líquidas de magnésio, potássio e sódio para o ganho de peso vivo foram obtidas dividindo-se as exigências líquidas para o ganho de peso corpo vazio (Tabela 5) pelo fator 1,25. Para as exigências líquidas de mantença, foram utilizados os valores propostos pelo ARC (1980).

Para o cálculo das exigências dietéticas, foram utilizados os valores de disponibilidade dos minerais preconizados pelo ARC (1980). As estimativas líquidas e dietéticas de magnésio, potássio e sódio são apresentadas nas Tabelas 6 a 10.

TABELA 5 - Estimativa da concentração de magnésio, potássio e sódio corporal do ganho em peso de corpo vazio de cordeiros SI e mestiços BE, IF e TE em função do peso corporal vazio.

\begin{tabular}{|c|c|c|c|c|c|c|c|}
\hline \multirow{2}{*}{$\begin{array}{l}\text { PV } \\
(\mathrm{kg})\end{array}$} & \multirow{2}{*}{$\begin{array}{l}\text { PCV } \\
(\mathrm{kg})\end{array}$} & \multicolumn{6}{|c|}{ Nutriente (g / kg PCV) } \\
\hline & & $\begin{array}{l}\text { Mg } \\
\text { (SI) }\end{array}$ & $\begin{array}{c}\text { Mg } \\
(\mathbf{B E}, \mathrm{IF}, \mathrm{TE})\end{array}$ & $\begin{array}{l}\mathrm{Na} \\
(\mathrm{IF})\end{array}$ & $\begin{array}{c}\mathbf{K} \\
(\mathbf{I F})\end{array}$ & $\begin{array}{c}\mathrm{Na} \\
(\mathrm{SI}, \mathrm{BE}, \mathrm{TE})\end{array}$ & $\begin{array}{c}\text { K } \\
(\text { SI,BE,TE) }\end{array}$ \\
\hline 15 & 10,87 & 0,509 & 0,481 & 1,239 & 1,628 & 1,292 & 1,715 \\
\hline 20 & 15,10 & 0,500 & 0,468 & 1,150 & 1,538 & 1,200 & 1,606 \\
\hline 25 & 19,35 & 0,483 & 0,459 & 1,086 & 1,477 & 1,135 & 1,528 \\
\hline 30 & 23,60 & 0,470 & 0,451 & 1,038 & 1,430 & 1,086 & 1,469 \\
\hline 35 & 27,83 & 0,459 & 0,445 & 1,000 & 1,392 & 1,047 & 1,421 \\
\hline 40 & 33,92 & 0,447 & 0,439 & 0,956 & 1,348 & 1,002 & 1,366 \\
\hline 45 & 36,31 & 0,443 & 0,417 & 0,941 & 1,333 & 0,986 & 1,348 \\
\hline Média & & 0,473 & 0,451 & 1,058 & 1,449 & 1,106 & 1,493 \\
\hline ARC(1980) & & 0,41 & 0,41 & 1,1 & 1,8 & 1,1 & 1,8 \\
\hline
\end{tabular}


TABELA 6 - Estimativas das exigências líquidas e dietéticas de magnésio para mantença e ganho em peso vivo (g/animal/dia), em animais Santa Inês.

\begin{tabular}{ccccccccc}
\hline \multirow{2}{*}{$\begin{array}{c}\text { Peso vivo } \\
\text { (kg) }\end{array}$} & \multicolumn{7}{c}{ Ganho Diário (g) } \\
\cline { 2 - 9 } & \multicolumn{7}{c}{ Exigência Líquida } & \multicolumn{7}{c}{ (g Mg/animal/dia) } \\
\cline { 2 - 9 } & Mantença $^{\mathbf{1}}$ & $\mathbf{1 0 0}$ & $\mathbf{2 0 0}$ & $\mathbf{3 0 0}$ & Mantença $^{\mathbf{1}}$ & $\mathbf{1 0 0}$ & $\mathbf{2 0 0}$ & $\mathbf{3 0 0}$ \\
\hline 15 & 0,045 & 0,0407 & 0,0814 & 0,1221 & 0,265 & 0,2394 & 0,4788 & 0,7182 \\
20 & 0,060 & 0,0400 & 0,0800 & 0,1200 & 0,353 & 0,2352 & 0,4704 & 0,7056 \\
25 & 0,075 & 0,0386 & 0,0772 & 0,1158 & 0,441 & 0,2270 & 0,4540 & 0,6810 \\
30 & 0,090 & 0,0376 & 0,0752 & 0,1128 & 0,529 & 0,2211 & 0,4422 & 0,6633 \\
35 & 0,105 & 0,0367 & 0,0734 & 0,1101 & 0,618 & 0,2158 & 0,4316 & 0,6474 \\
40 & 0,120 & 0,0357 & 0,0714 & 0,1071 & 0,705 & 0,2100 & 0,4200 & 0,6300 \\
45 & 0,135 & 0,0354 & 0,0708 & 0,1062 & 0,794 & 0,2082 & 0,4164 & 0,6246 \\
\hline
\end{tabular}

1 Valor preconizado pelo ARC (1980)

TABELA 7 - Estimativas das exigências líquidas e dietéticas de magnésio para mantença e ganho em peso vivo (g/animal/dia), em animais BE,IF,TE.

\begin{tabular}{ccccccccc}
\hline \multirow{2}{*}{$\begin{array}{c}\text { Peso vivo } \\
\text { (kg) }\end{array}$} & \multicolumn{7}{c}{ Ganho Diário (g) } \\
\cline { 2 - 10 } & \multicolumn{7}{c}{ Exigência Líquida } & \multicolumn{7}{c}{ (g Mg/animal/dia) } \\
\hline & Mantença $^{\mathbf{1}}$ & $\mathbf{1 0 0}$ & $\mathbf{2 0 0}$ & $\mathbf{3 0 0}$ & Mantença $^{\mathbf{1}}$ & $\mathbf{1 0 0}$ & $\mathbf{2 0 0}$ & $\mathbf{3 0 0}$ \\
\hline 15 & 0,045 & 0,0384 & 0,0768 & 0,1152 & 0,265 & 0,2258 & 0,4516 & 0,6774 \\
20 & 0,060 & 0,0374 & 0,0748 & 0,1122 & 0,353 & 0,2200 & 0,4400 & 0,6600 \\
25 & 0,075 & 0,0367 & 0,0734 & 0,1101 & 0,441 & 0,2158 & 0,4316 & 0,6474 \\
30 & 0,090 & 0,0360 & 0,0720 & 0,1080 & 0,529 & 0,2117 & 0,4234 & 0,6351 \\
35 & 0,105 & 0,0356 & 0,0712 & 0,1068 & 0,618 & 0,2094 & 0,4188 & 0,6282 \\
40 & 0,120 & 0,0351 & 0,0702 & 0,1053 & 0,705 & 0,2064 & 0,4128 & 0,6192 \\
45 & 0,135 & 0,0333 & 0,0666 & 0,0999 & 0,794 & 0,1958 & 0,3916 & 0,5874 \\
\hline
\end{tabular}

1Valor preconizado pelo ARC (1980) 
TABELA 8 - Estimativas das exigências líquidas e dietéticas de potássio para a mantença e ganho em peso vivo (g/animal/dia),em ovinos IF, SI,BE,TE.

\begin{tabular}{ccccccccc}
\hline \multirow{2}{*}{\begin{tabular}{c} 
Peso vivo $\begin{array}{c}\text { Ganho Diário (g) } \\
\text { (kg) }\end{array}$ \\
\cline { 2 - 10 }
\end{tabular}} & \multicolumn{7}{c}{ (g K/animal/dia) } \\
\cline { 2 - 10 } & \multicolumn{7}{c}{ Exigência Líquida e Dietética (IF) } & \multicolumn{7}{c}{ Exigência Líquida e Dietética ( SI,BE,TE) } \\
\hline & Mantença $^{1}$ & $\mathbf{1 0 0}$ & $\mathbf{2 0 0}$ & $\mathbf{3 0 0}$ & Mantença $^{1}$ & $\mathbf{1 0 0}$ & $\mathbf{2 0 0}$ & $\mathbf{3 0 0}$ \\
\hline 15 & 1,566 & 0,130 & 0,258 & 0,387 & 1,566 & 0,137 & 0,274 & 0,411 \\
20 & 1,790 & 0,123 & 0,246 & 0,369 & 1,790 & 0,128 & 0,256 & 0,384 \\
25 & 2,112 & 0,118 & 0,236 & 0,354 & 2,112 & 0,122 & 0,244 & 0,366 \\
30 & 2,435 & 0,114 & 0,228 & 0,342 & 2,435 & 0,117 & 0,234 & 0,351 \\
35 & 2,657 & 0,111 & 0,222 & 0,333 & 2,657 & 0,113 & 0,226 & 0,339 \\
40 & 2,893 & 0,107 & 0,214 & 0,321 & 2,893 & 0,109 & 0,218 & 0,327 \\
45 & 3,150 & 0,106 & 0,212 & 0,318 & 3,150 & 0,107 & 0,214 & 0,321 \\
\hline
\end{tabular}

1'Valor preconizado pelo ARC (1980)

TABELA 9 - Estimativas das exigências líquidas e dietéticas de sódio para a mantença e ganho em peso vivo (g/animal/dia),em ovinos IF.

\begin{tabular}{|c|c|c|c|c|c|c|c|c|}
\hline \multirow{4}{*}{$\begin{array}{c}\text { Peso vivo } \\
\text { (kg) }\end{array}$} & \multicolumn{8}{|c|}{ Ganho Diário (g) } \\
\hline & \multicolumn{8}{|c|}{ (g Na/animal/dia) } \\
\hline & \multicolumn{3}{|c|}{ Exigência Líquida } & \multicolumn{5}{|c|}{ Exigência Dietética } \\
\hline & Mantença $^{1}$ & 100 & 200 & 300 & Mantença $^{1}$ & 100 & 200 & 300 \\
\hline 15 & 0,387 & 0,099 & 0,198 & 0,297 & 0,425 & 0,108 & 0,216 & 0,324 \\
\hline 20 & 0,516 & 0,092 & 0,184 & 0,276 & 0,567 & 0,101 & 0,202 & 0,303 \\
\hline 25 & 0,645 & 0,086 & 0,172 & 0,258 & 0,708 & 0,094 & 0,188 & 0,282 \\
\hline 30 & 0,774 & 0,083 & 0,166 & 0,249 & 0,850 & 0,091 & 0,182 & 0,273 \\
\hline 35 & 0,903 & 0,080 & 0,160 & 0,240 & 0,992 & 0,087 & 0,174 & 0,261 \\
\hline 40 & 1,032 & 0,076 & 0,152 & 0,228 & 1,134 & 0,083 & 1,166 & 0,249 \\
\hline 45 & 1,061 & 0,075 & 0,150 & 0,225 & 1,275 & 0,082 & 0,164 & 0,246 \\
\hline
\end{tabular}

1'Valor preconizado pelo ARC (1980) 
TABELA 10 - Estimativas das exigências líquidas e dietéticas de sódio para a mantença e ganho em peso vivo (g/animal/dia), em ovinos SI, BE, TE.

\begin{tabular}{ccccccccc}
\hline \multirow{2}{*}{$\begin{array}{c}\text { Peso vivo } \\
\text { (kg) }\end{array}$} & \multicolumn{7}{c}{ Ganho Diário (g) } \\
\cline { 2 - 9 } & \multicolumn{7}{c}{ Exigência Líquida } \\
\cline { 2 - 10 } & \multicolumn{7}{c}{ (g Na/animal/dia) } \\
\hline & Mantença $^{1}$ & $\mathbf{1 0 0}$ & $\mathbf{2 0 0}$ & $\mathbf{3 0 0}$ & Mantença $^{1}$ & $\mathbf{1 0 0}$ & $\mathbf{2 0 0}$ & $\mathbf{3 0 0}$ \\
\hline 15 & 0,387 & 0,103 & 0,206 & 0,309 & 0,425 & 0,113 & 0,226 & 0,339 \\
20 & 0,516 & 0,096 & 0,192 & 0,288 & 0,567 & 0,105 & 0,210 & 0,315 \\
25 & 0,645 & 0,090 & 0,180 & 0,270 & 0,708 & 0,098 & 0,196 & 0,294 \\
30 & 0,774 & 0,086 & 0,172 & 0,258 & 0,850 & 0,094 & 0,188 & 0,282 \\
35 & 0,903 & 0,083 & 0,166 & 0,249 & 0,992 & 0,091 & 0,182 & 0,273 \\
40 & 1,032 & 0,080 & 0,160 & 0,240 & 1,134 & 0,087 & 0,174 & 0,261 \\
45 & 1,061 & 0,078 & 0,156 & 0,234 & 1,275 & 0,085 & 0,170 & 0,255 \\
\hline
\end{tabular}

${ }^{1}$ Valor preconizado pelo ARC (1980).

Comparando as exigências líquidas de magnésio obtidas nesta pesquisa com os valores propostos pelo ARC (1980), observa-se que essas são semelhantes para os animais SI com $15 \mathrm{~kg}$ de peso vivo e $17,14 \%$ menores para animais com $45 \mathrm{~kg}$ de peso vivo. Já para os animais mestiços BE, IF, TE, observa-se que essas são aproximadamente $7,89 \%$ inferiores para animais com 15 $\mathrm{kg}$ e $24,24 \%$ para animais com $45 \mathrm{~kg}$ de peso vivo.

Analisando o potássio, as exigências líquidas obtidas neste trabalho são $31,38 \%$ inferiores para cordeiros com $15 \mathrm{~kg}$ de PV para os animais SI, BE, TE, quando comparadas com os valores preconizados pelo ARC (1980). Já para o sódio, as estimativas foram inferiores em 6,79\% para animais com $15 \mathrm{~kg}$ de PV e $41,02 \%$ para animais com $45 \mathrm{~kg}$ de peso vivo, para os animais SI, BE e TE.

É preciso ressaltar que a composição corporal e, conseqüentemente, as exigências de $\mathrm{Mg}, \mathrm{Na}$ e $\mathrm{K}$ irão variar, principalmente em função da proporção de ossos, músculos e gordura corporal, e qualquer fator que afete essas proporções irá afetar as exigências desses minerais.

Dessa forma, os valores de exigências de ganho de peso preconizados pelo ARC (1980) devem ser adotados com certas restrições, uma vez que essa tabela foi estabelecida utilizando animais diferentes dos nossos e sob condições climáticas diversas.

\section{CONCLUSÕES}

a) Existem diferenças na composição corporal de magnésio, sódio e potássio entre ovinos SI, ovinos mestiços (IF X SI) e os demais grupos genéticos.

b) O grupo racial deverá ser considerado ao estimar a composição corporal e as exigências nutricionais.

\section{REFERÊNCIAS BIBLIOGRÁFICAS}

AGRICULTURAL RESEARCH COUNCIL. The nutrient reguirements of farm livestock. London, 1980. $351 \mathrm{p}$.

ANNENKOV, B. N. Mineral feeding of sheep. In: GEORGIEVSKII, V. I.; ANNENKOV, B. N.; SAMOKHIN, V. I. Mineral nutrition of animals. London: Butterworths, 1982. p. 331-354.

GERASEEV, L. C.; PEREZ, J. R. O.; RESENDE, K. T.; PAIVA, P. C. A.; PRADO, O. V. Composição corporal e exigências nutricionais de magnésio, potássio e sódio de cordeiros Santa Inês em crescimento dos $25 \mathrm{~kg}$ aos $35 \mathrm{~kg}$ de peso vivo. Ciência e Agrotecnologia, Lavras, v. 25, n. 2, p. 386-395, mar./abr. 2001. 
GRACE, N. D. Amounsts and distribution of mineral elements associated with fleece-free empty body weight gaisns in the grazing sheep. New Zealand Journal of Agricultural Research, Wellington, v. 26, p. 59-70, 1983.

MCDOWELL, L. R. Minerais para ruminantes sob pastejo em regiões tropicais: enfatizando o Brasil. Gainesville: Universidade da Flórida, 1999. p. 93.

NATIONAL RESEARCH COUNCIL. Nutrient regueriments of domestic animals: nutríent requeriments of sheep. Washington, 1985. $99 \mathrm{p}$.

SILVA, J. F. C. Exigências de macroelementos inorgânicos para bovinos: o sistema ARC/AFRC e a experiência no Brasil. In: SIMPÓSIO INTERNACIONAL SOBRE EXIGÊNCIAS NUTRICIONAIS DE RUMINANTES, 1995, Viçosa. Anais... Viçosa: [s.n.], 1995. p. 467-504.

SNEDECOR, G. W.; COCHRAN, W. G. Statistical methods. 6. ed. Iowa: The Iowa State University, 1967. $593 \mathrm{p}$.

TRINDADE, I. A. C. M.; RESENDE, T. K.; SILVA, A. M. A.; SILVA SOBRINHO, A. G.; PÉREZ, J. R. O. Composição corporal e exigências líquidas de Magnésio, Potássio e Sódio de cordeiros Lanados e Deslanados. In: SOCIEDADE BRASILEIRA DE ZOOTECNIA, 37., 2000, Viçosa. Anais... Viçosa: SBZ, 2000. p. 364 